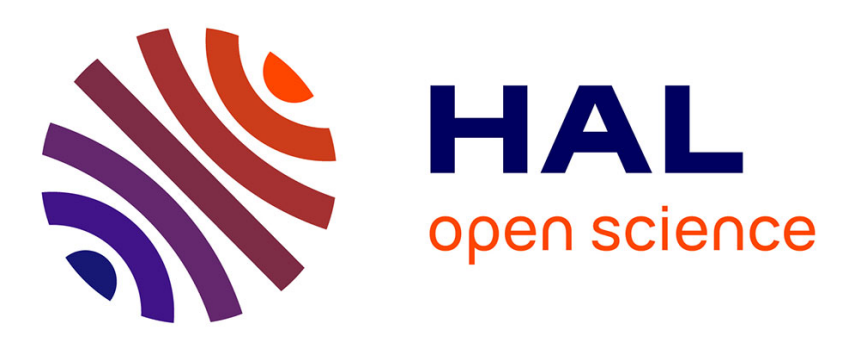

\title{
On the Complexity of an Accurate and Precise Performance Evaluation of Wireless Networks Using Simulations
}

Elyes Ben Hamida, Guillaume Chelius, Jean-Marie Gorce

\section{- To cite this version:}

Elyes Ben Hamida, Guillaume Chelius, Jean-Marie Gorce. On the Complexity of an Accurate and Precise Performance Evaluation of Wireless Networks Using Simulations. 11th ACM/IEEE international symposium on Modeling, Analysis and Simulation of Wireless and Mobile Systems (MSWIM 2008), IEEE / ACM, Oct 2008, Vancouver, Canada. pp.395-402, 10.1145/1454503.1454570 . inria00384082

\section{HAL Id: inria-00384082 \\ https://hal.inria.fr/inria-00384082}

Submitted on 14 May 2009

HAL is a multi-disciplinary open access archive for the deposit and dissemination of scientific research documents, whether they are published or not. The documents may come from teaching and research institutions in France or abroad, or from public or private research centers.
L'archive ouverte pluridisciplinaire HAL, est destinée au dépôt et à la diffusion de documents scientifiques de niveau recherche, publiés ou non, émanant des établissements d'enseignement et de recherche français ou étrangers, des laboratoires publics ou privés. 


\title{
On the Complexity of an Accurate and Precise Performance Evaluation of Wireless Networks Using Simulations
}

\author{
Elyes Ben Hamida, Guillaume Chelius, and Jean-Marie Gorce \\ Université de Lyon, INRIA \\ INSA-Lyon, CITI, F-69621, France \\ elyes.ben-hamida@insa-lyon.fr, guillaume.chelius@inria.fr, \\ jean-marie.gorce@insa-lyon.fr
}

\begin{abstract}
In wireless multi-hop networks, there is a growing need for the performance evaluation of protocols and distributed applications. Due to the high complexity of analytical models and the difficulty of setting up large scale testbeds, simulations are generally considered as the most convenient methodology for evaluating the performance of wireless systems. However, evaluating a protocol can be a tedious task as it depends on several factors including the physical layer modeling and the dimensioning of protocol or environment parameters. As a consequence inferring an overall performance for a protocol is a real issue. In this paper, we highlight some main factors that may affect the accuracy of the performance evaluation of protocols using simulations. First, we focus on the physical layer (PHY) modeling which represent a key point for the sake of realism and confidence. Second, we discuss the impact of some protocol parameters on the behavior of the evaluated protocols.
\end{abstract}

\section{Categories and Subject Descriptors}

I.6.7 [Simulation Support Systems]: Environments; C.2.1 [Network Architecture and Design]: Wireless communication

\section{General Terms}

Performance

\section{Keywords}

Wireless networks, physical layer modeling, performance analysis

\section{INTRODUCTION}

Due to the technological advances in miniaturization, low-power circuit design and efficient wireless capability, mobile ad hoc networks (MANET) have emerged as a promising technology with numerous military and civil applications, such as emergency operations, disaster recoveries, tactical surveillance, etc. Such networks are composed by a collection of wireless nodes that can

Permission to make digital or hard copies of all or part of this work for personal or classroom use is granted without fee provided that copies are not made or distributed for profit or commercial advantage and that copies bear this notice and the full citation on the first page. To copy otherwise, to republish, to post on servers or to redistribute to lists, requires prior specific permission and/or a fee.

MSWiM'08, October 27-31, 2008, Vancouver, BC, Canada.

Copyright 2008 ACM 978-1-60558-235-1/08/10 ...\$5.00. dynamically self-organize to form a network without using any pre-existing infrastructure. More recently, there have been growing research interest in large-scale and autonomous wireless sensor networks (WSN), where a hundreds or a thousands of energyconstrained sensors are deployed over an area of interest to provide connectivity and monitor ambient conditions in the surrounding environment. Numerous applications are envisaged for wireless sensor networks such as, weather monitoring, fire detection, target tracking, etc. In wireless multi-hop networks, there is a growing need for the performance evaluation of networking protocols. Contrarily to the reliable physical medium of wired networks, wireless systems rely on a radio medium which is prone to failure. In such a context, the medium is shared by several nodes increasing packet loss due to collision and interference. In particular, interference is a major limiting factor for wireless communication. This issue makes it harder the performance evaluation of wireless networking protocols.

Three main approaches are generally adopted for the performance evaluation of networking protocols: analytical analysis, experimentation and simulation. Due to the high complexity of wireless communications, analytical analysis are often based on unrealistic assumptions (e.g., node synchronisation, ideal MAC layer, homogeneous location model, symmetric radio links, etc.) and inaccurate physical layer (PHY) models. An example of inaccurate PHY modeling is the famous disk model which has been widely used to model the radio range of wireless nodes where the interference is generally not taken into account. In addition, theoretical analysis always focuses on a given layer, ignoring the other network layers. Concerning the experimentation approach, it can provide valuable insight into the behavior of protocols in wireless environments. However, setting up large scale testbeds is a tedious task and is not always feasible. Moreover, the obtained results are strongly correlated to the surrounding environment and are not easily reproducible.

For these reasons, the use of simulations is generally considered as the most convenient methodology to analyse the performance of protocols and distributed applications. Nonetheless, the complexity of the physical phenomena constituting the radio medium introduces a tradeoff between accuracy and computational cost in wireless network simulation. Several wireless network simulators have been proposed in the last years. Examples are NS-2, GloMoSim [2], JiST/SWANS [3], GTSNetS [10], OMNeT++, OPNET, etc. They all provide an advanced and complete simulation environment to investigate and evaluate networking protocols and wireless systems. However, the complexity of the wireless physical layer (PHY) leads to implementation choices during the simulators 
design. As a consequence, the PHY simulation accuracy varies drastically from one simulator to another. In particular, interference management is probably the point where current simulators differ the most largely. The reason that generally justifies a low accuracy is performance. Moreover, some protocol and environment parameters are generally tuned arbitrarily during the performance evaluation of high level protocols. In this context, it is hard to obtain a representative and an overall performance evaluation of protocols. In fact, several previous publications have shown that the behavior of wireless networking protocols may radically differ from a simulation environment to another one $[4,8,15]$.

In this paper, we highlight the main factors that may affect the accuracy of the simulation results. First, we focus on the physical layer modeling which represents a key point for the sake of realism and confidence. The question we raise is: what is the real impact of the physical layer modeling on the behavior of the evaluated high level protocols ?. To analyse this question, we present the main physical modeling issues, including the radio range modeling, the radio link modeling and the interference modeling. Then, we review the PHY models implemented in common simulators and we use the WSNet [5] simulation environment to analyse the impact of the PHY modeling on the accuracy of the performance evaluation of high level protocols. Finally, we investigate the impact of some protocol and environment parameters on the performance evaluation of application-layer protocols. The second question we raise is: what is the impact of the dimensioning of these protocols on the global performance accuracy?. We focus on the dimensioning of a hello protocol and the battery parameters.

The remainder of this paper is organized as follows. In Section 2 we discuss the related work in modeling of wireless systems using simulation model. In Section 3, we describe the main physical layer modeling issues and we review the PHY models of common simulators. Then, in Section 4, we analyze qualitatively the impact of the PHY modeling on the behavior of a layer-3 protocol. Next, in Section 5, we investigate the impact of some protocol and environment parameters on the global performance of protocols. Finally, we conclude in Section 6.

\section{BACKGROUND}

Wireless network simulators. Numerous wireless network simulators have been developed and are concurrently used in the academic research world. The $\mathbf{N S}-\mathbf{2}$ network simulator is one of the most popular environment for wired and wireless network simulations. NS-2 is developed in C++ and uses OTcl for scripting and configuration, however it suffers from a limited scalability though some recent optimizations have been proposed to support simulations of a few thousand nodes [9]. GloMoSim [2] is a simulation environment based on a C-derived language, called Parsec, which supports the sequential and parallel execution of discreteevent simulations. Thanks to parallelization, GloMoSim was shown to scale up to 10,000 nodes. The JiST/SWANS [3] is a scalable java-based discrete event simulation for wireless networks. It was shown that JiST/SWANS outperforms NS-2 and GloMoSim in terms of scalability and memory usage. The Georgia Tech Network Simulator, GTNeTS [10], is a C++ object-oriented simulation environment dedicated to the simulation of wireless sensor networks. GTSNeTS claims to scale to networks of several hundred thousands of nodes.

More recently, we have proposed the WSNet [5] simulation framework. The main goal of WSNet is to provide a modular architecture which eases the development and the addition of new simulation models. Moreover, WSNet offers a wide range of PHY layer modeling, starting from a basic perfect $\mathrm{PHY}$ layer to a very precise one.
In this paper, we choose to use $\mathrm{WSNet}^{1}$ for our performance evaluation analysis as none of the existing simulator offer a sufficient diversity in PHY models.

Modeling and simulation of wireless networks. The literature in the context of modeling of wireless systems provides a lot of papers analyzing the accuracy of the simulation results. In [4], the authors investigate the accuracy of three popular simulators (NS2, GloMoSim and OPNET). Through the simulation of a basic flooding algorithm, they show a significant divergence in the obtained results between the simulators. The major reason for this issue is the PHY layer modeling which is implemented differently from a simulator to another. Indeed, in [15], the physical layer models of these three simulators is presented in detail, and the authors discuss some PHY layer factors which are relevant to the performance evaluation of protocols. In [8], the authors discuss the effects of detail in wireless simulation. They show how the performance evaluation of protocols can vary when tuning the level of detail. They suggest to adapt the level of detail required by a given question. In [16], the authors describe the impact of using detailed models for battery, processor power consumption and traffic models on the behavior of protocols.

Contribution. Our contribution is twofold. First, we provide an in-depth analysis of the PHY layer modeling and we show, through extensive simulations, its impact on the accuracy of the performance evaluation of high level protocols. Second, we investigate the impact of the dimensioning of some protocol and environment parameters on the global performance of the protocol.

\section{PHYSICAL LAYER MODELING ISSUES}

For the sake of realism and confidence in simulation results, an accurate PHY modeling is a key point. In analytical studies as in simulations, the disk model has long prevailed. It relies on a set of strong assumptions:

$$
\begin{array}{ll}
\text { time stationarity : } & l_{i j}(t)=l_{i j} \\
\text { independence : } & l_{i j}=f\left(x_{i}, x_{j}\right) \\
\text { switched link (on/off) : } & l_{i j} \in\{0,1\} \\
\text { symmetry : } & l_{i j}=l_{j i} \\
\text { isotropy : } & l_{i j}=f\left(x_{i}, d_{i j}\right) \\
\text { homogeneity : } & l_{i j}=f\left(d_{i j}\right)
\end{array}
$$

where $l_{i j}$ refers to the radio link between nodes $i$ and $j$ and $d_{i j}$ to the geometric distance between $i$ and $j$..

The disk model provides the radio network with three axioms: the radio range is constant, the radio link is switched, and the network is interference free. If the asset of this model holds in its simplicity for both theoretical studies and simulations, the resulting simulations are far from realistic. Nonetheless, improving this model is not a trivial task as a hard tradeoff between complexity and realism holds. Basically, this model can be improved by relaxing either of the three previously stated axioms, as discussed below.

\subsection{Radio range modeling}

The range of a radio system is based upon the definition of a signal to noise ratio (SNR) threshold noted $\bar{\gamma}_{l i m}$. If the system is interference free, the range is a constant and the radio link is defined

\footnotetext{
${ }^{1}$ WSNet is available at: http://wsnet.gforge.inria.fr.
} 
by:

$$
\begin{aligned}
& l_{i j}: \Omega^{2} \mapsto B=\{0,1\} \\
& \left(x_{i}, x_{j}\right) \mapsto l\left(x_{i}, x_{j}\right)= \begin{cases}1 & \text { if } \bar{\gamma}_{i j} \geq \bar{\gamma}_{l i m} \\
0 & \text { else }\end{cases}
\end{aligned}
$$

where the SNR $\bar{\gamma}_{i j}$ is given by: $\bar{\gamma}_{i j}=h_{i j} \cdot \frac{P_{i}}{N_{j}}$, where $h_{i j}$ is the path-loss and $P_{i}$ and $N_{j}$ are the transmission power and the noise level respectively.

The transceiver properties. They are the transmission power $P_{i}$, the noise level $N_{i}$, the antenna gain and its radiation pattern $g_{i}(\theta, \phi)$. Variations of $P_{i}, N_{i}$ or antenna gains affect the spatial homogeneity assumption (and so far the symmetry), which means that all nodes not further have the same range. Note that a non-uniform noise level $N_{j}$ is highly probable for low cost small radio systems. The isotropy which is not statistically affected by these parameters does not further hold if the radiation patterns of the antennas are introduced according to: $h_{i j}=g\left(x_{i}, x_{j}\right) \cdot g_{i}\left(\theta_{i j}, \phi_{i j}\right) \cdot g_{j}\left(\theta_{j i}, \phi_{j i}\right)$, where $g\left(x_{i}, x_{j}\right)$ is the propagation path-loss. It should be noted that $3 \mathrm{D}$ radiation pattern and $3 \mathrm{D}$ distribution of radio systems is pertinent for the simulation of small indoor environments for example.

Propagation models. The simplest model refers to the line of sight (LOS) scenario but in urban and indoor environments, more complicated scenarios occur due to shadowing and multiple paths. Two complementary approaches can be used to deal with propagation. The former approach relies on a deterministic modeling of the wave propagation and provides fine simulations of any environment. The most usual algorithms are ray-tracing based [1] but discrete methods have been also proposed [6]. The high accuracy of these methods is definitely balanced by their high computational cost. Another limitation of purely deterministic models is that simulating one real environment is often too specific. Thus, the later approach relies on a statistical description complementing the deterministic model. A stochastic variable $\mathbf{s}_{i j}$ is then introduced in the propagation path-loss to handle shadowing: $\mathbf{g}_{i j}=g_{i j} \cdot \mathbf{s}_{i j}$.

The most usual model is the log-normal shadowing. A very challenging issue with shadowing is to introduce a spatial correlation between radio links which is not found in current simulators. Thus, $\mathbf{s}_{i j}$ should be a spatial correlated stochastic process constant in time; it is not the case in some works as shadowing is often confused with fading. Fading refers to SNR time variations due to multi-path interference. It has a leading role in wireless communications and is introduced also as a stochastic variable $\mathbf{f}_{i j}$ :

$$
\mathbf{g}_{i j}(t)=g_{i j} \cdot \mathbf{s}_{i j} \cdot \mathbf{f}_{i j}(t)
$$

This late variable is not spatially correlated as it relies on small scale phenomenon. Meanwhile, it is a time variant parameter. Considering its temporal correlation may be highly relevant [11] and represents another challenge for wireless simulators.

\subsection{Radio link modeling}

A frame error rate (FER) as a function of the mean SNR can substitutes for the SNR threshold of Equation 7. It derives from the bit error rate (BER) function which itself relies on the radio interface properties. $l_{i j}$ then relates to the probability of a successful transmission. Some theoretical asymptotic expressions are well-known for various modulation techniques [17]. However, the exact derivation at low SNR is sometimes not straightforward more specifically in the case of fading channels, which can be introduced at this level with:

$$
P_{s}(E \mid \bar{\gamma})=\int_{0}^{\infty} P_{s}(E \mid \gamma) \cdot f_{\gamma}(\gamma \mid \bar{\gamma}) \cdot d \gamma_{s}
$$

where $f_{\gamma}(\gamma \mid \bar{\gamma})$ stands for the instantaneous SNR distribution due to fading. It can be also of great importance to considering channel coding. For instance, bloc coding can be introduced thanks to bounding the code word error probability. Last but not least, the radio link can be more complex if the pulse time-spreading due to multi-path lies beyond the symbol period. In this case, the impulse response should be considered.

\subsection{Interference modeling}

Interference disturbs the packet reception at the physical layer. It appears as a crucial point in PHY simulations as final results can be strongly influenced by the interference model. As we will see in SubSection 3.4, interference management is probably the point where current simulators differ the most largely. Sources of interference include nodes operating in the same frequency band or in different frequencies. The first type of interference is known as co-channel interference, while the latter is termed adjacent channel interference.

The most efficient approach for introducing interference consists in replacing the SNR by a signal to interference plus noise ratio, SINR, which can be derived according to:

$$
\bar{\gamma}_{i j}=h_{i j} \cdot \frac{P_{i}}{N_{j}+\sum_{k \neq i, j} h_{k j} \cdot P_{k}}
$$

The proper derivation of the SINR requires the knowledge, at a given time, of all the signals which are concurrently received at a given receiver. To be exhaustive, it should be noted that non linear receivers (with multi-user detection for instance) can outperform classical receivers in the presence of interference. In this case, it would be necessary to compute the FER not from the general SINR, but rather from the vector of received powers at each node.

\subsection{Physical modeling in common simulators}

Several wireless network simulators have been proposed in the last years. They all provide an advanced and complete simulation environment to investigate and evaluate networking protocols and wireless systems. However, the complexity of the wireless physical layer (PHY) leads to implementation choices during the simulators design. As a consequence, the PHY simulation accuracy varies drastically from one simulator to another, as shown on Table 1. Interference modeling, which is one of the most crucial aspects for cross-layer and protocol studies, is probably the point where current simulators differ the most largely.

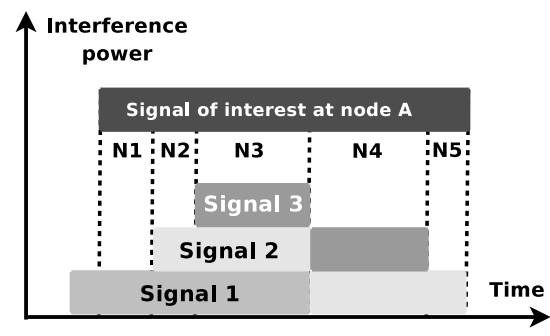

Figure 1: Strategies for the SINR computation.

The first step toward interference evaluation is to identify which signals are interfering with each other in order to assess the terms in the denominator of eq. 9, on the basis of timing considerations only. This set of interfering signals can be very large for large scale simulations. As a consequence, various simulators rather limit the range at which any signal can propagate and thus can interfere. In other words, disregarding the radio range model effectively used for the 


\begin{tabular}{|c|c|c|c|c|c|c|c|}
\hline \multirow{2}{*}{$\begin{array}{c}\text { Simulation } \\
\text { environments }\end{array}$} & \multicolumn{3}{|c|}{ Radio range modeling } & \multicolumn{2}{|c|}{ Radio link modeling } & \multicolumn{2}{|c|}{ Interference modeling } \\
\hline & pathloss & shadowing & fading & link model & modulation & model & SINR computation \\
\hline NS-2 & free space, two-ray & log-normal & "rician, rayleigh & threshold & - & limited & strongest signal \\
\hline GloMoSim [2] & free-space, two-ray & log-normal & rician, rayleigh & threshold, BER & BPSK, QPSK & limited & adaptive \\
\hline JiST/SWANS [3] & free-space, two-ray & - & rician, rayleigh & threshold, BER & BPSK & limited & cumulative \\
\hline GTSNetS [10] & free-space, two-ray & - & - & threshold & - & limited & strongest signal \\
\hline WSNet [5] & free-space, two-ray & log-normal & rician, rayleigh & threshold, BER & $\begin{array}{c}\text { BPSK, STEP, } \\
\text { OQPSK, FSCK }\end{array}$ & $\begin{array}{l}\text { limited, } \\
\text { full }\end{array}$ & $\begin{array}{l}\text { adaptive, } \\
\text { cumulative }\end{array}$ \\
\hline
\end{tabular}

Table 1: PHY layer modeling in common simulation environments: NS 2.31, JiST/SWANS 1.06, GloMoSim 2.03, GTSNetS and WSNet 2.0.

received signal strength computation, the simulator does not generate receptions at nodes further than a given range from the source. Consequently, the considered source cannot induce interference at nodes further than this range. This optimization is called Limited interference model as opposed to the Full interference model. It privileges scalability at the cost of accuracy. This optimization is implemented in NS-2, JiST/SWANS, GloMoSim, etc.

Regarding the SINR computation, several strategies have been investigated and implemented in existing simulators, as shown on Table 1. They are all variations of eq. 9 regarding timing granularity. They induce a varying level of realism, precision but also complexity. Three main approaches, for the SINR computation, are adopted: (i) strongest signal: the noise value considered for the SINR computation is the power of the strongest interfering signal (i.e., $N 3$ on Figure 1); (ii) cumulative: the noise value considered for the SINR computation is the cumulative power of the signals that interfere with the considered signal at reception time (i.e., $N 5$ on Figure 1); and (ii) adaptive: several SINR values are computed for the packet; whenever the set of interfering signals changes, a new SINR value is computed. The noise value considered for each SINR computation is the cumulative power of all concurrent interfering signals (i.e., from $N 1$ to $N 5$ on Figure 1).

It is obvious that these interference models offer different levels of complexity, accuracy or realism. In the next section, we evaluate, using the WSNet simulation framework, the impact of these on the behavior of high level protocols.

\section{IMPACT OF THE PHY MODELING ON LAYER-3 PROTOCOLS}

This section quantifies the impact of the physical modeling on the behavior of a high level protocol. First, we present the simulation environment and the assumptions. Then, we investigate the impact of the radio range modeling as well as the radio link and the interference modeling on a hello protocol using WSNet. We choose to study a hello protocol as it represents an important network service on which several protocols are based, e.g., routing and self-organizing protocols.

\subsection{Assumptions}

We consider 500 static nodes, randomly deployed in a $200 \times$ 200 area, running a basic hello protocol. Using this protocol, each node can be in two states: listening or talking. These states occur inside a timeframe of duration $w$. In each occurrence of $w$ (a.k.a., in each round or timeframe of the hello protocol), a node picks randomly an offset $t_{i}$, such that $t_{i} \in[0, w-\tau]$. The hello packet is then transmitted at $t_{i}$ during $\tau$. The hello packet transmission is performed without any carrier sensing nor clear channel assessment. Each node transmits only one hello packet per timeframe and keeps listening to the medium during the rest of the frame. Upon the reception of a hello packet, the transmitter is said to be discovered by the receiver which adds an entry in its neighbor table.
This entry contains, in general, the neighbor identifier and a timeout to remove old entries associated to mobile or dead nodes. The neighbor table is then used by layer- 3 protocols for determining, for example, the next hop during the routing process.

For our analysis, we assume a timeframe, $w$, of duration $500 \mathrm{~ms}$, and that each node emits one hello packet per round $(100 \mathrm{~B} / \mathrm{s})$ through an IEEE 802.15.4 868Mhz compliant radio with a transmission power of $0 \mathrm{dBm}$ and a radio sensitivity threshold of -92 $d B m$. The hello protocol is then evaluated according to the following four metrics: (i) the number of discovered neighbors: which is the average number of discovered neighbors at each round of the hello protocol; (ii) the distance from the farthest discovered neighbor: which is the maximal distance from which a node have been discovered; (iii) the number of connex components: which reflects the network connectivity. The more the number of connex components increases, the more network connectivity is reduced; and (iv) the link success probability: which is the probability of successful transmission according to the distance between the transmitter and the receiver. These metrics are evaluated at the end of each round, and for a node located at the center of the simulation area in order to avoid the edge effects. We performed the same set of simulations with various PHY models. The simulation results are averaged over 30 runs with a $95 \%$ confidence interval.

\subsection{Effects of the radio range modeling}

We first start by evaluating the impact of the radio range modeling on the behavior of the hello protocol. The interference modeling is not yet taken into account. We consider ( $i$ ) a free-space propagation model with a pathloss of 2; (ii) a log-normal shadowing model with a standard deviation of $4 d B$ and a close-in reference distance of $1 \mathrm{~m}$; and (iii) a rayleigh block-fading model. At this time the shadowing model does not include spatial correlation and the fading is uncorrelated between two frames. These radio range models are compared according to the above metrics. The average number of discovered neighbors, the distance from the farthest discovered neighbor and the number of connex components are shown on Figure 2 in function of the time, while the distribution of the link success probability is drawn on Figure 3. This probability represents the probability that a link exists between a transmitter and a receiver in function of the distance between them.

We notice on Figure 2-(a), that the average number of discovered neighbors, at each round, is almost the same for the three models; however, we observe that the average number of connex components is slightly different, as shown on Figure 2-(c). Indeed, the pathloss model induces a network which is less connected (i.e., a higher number of connex components) than the one obtained with the fading and the shadowing models. This fact can be explained by the log-normal random variable, introduced in the shadowing and fading models, which allows longer communication range than the pathloss model, as shown on Figure 2-(b), and thus the network connectivity is improved.

Considering the link success probability, shown on Figure 3, we 


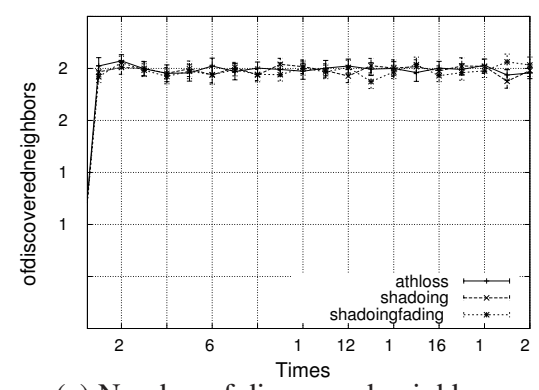

(a) Number of discovered neighbors.

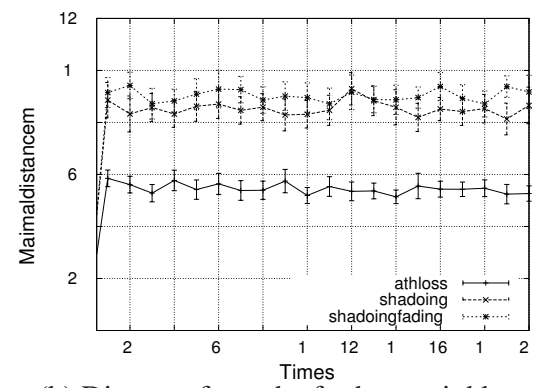

(b) Distance from the farthest neighbor

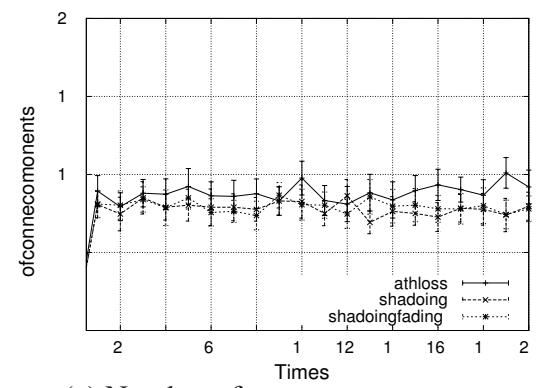

(c) Number of connex components.

Figure 2: Impact of the radio range modeling on the behavior of a hello protocol (without interference modeling).

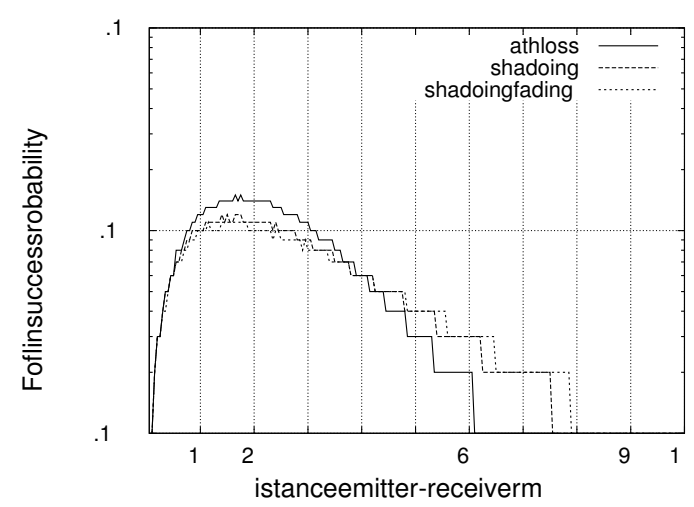

Figure 3: Impact of the radio range modeling on the link success probability.

observe that the link probability decreases in function of the distance between the emitter and the receiver, and that the shadowing and fading models present a higher link success probability for higher distance. These results show that the radio range modeling impacts strongly the network connectivity as well as the link success probability. As most of layer-3 protocols exploit the graph of connectivity, obtained by the hello protocol, it is thus important to use an adequate radio range model for the performance evaluation of networking protocols.

\subsection{Effects of the radio link modeling}

We now evaluate the impact of the radio link modeling on the behavior of the hello protocol. Two radio link models are considered: the threshold-based and the BER-based models. In the thresholdbased model, a packet is correctly received iff the signal to noise ratio (SNR) is higher than a given threshold. This model is used, for example, in NS-2, GTSNetS [10], etc. In the BER-based model, the packet reception depends on the frame error rate (FER) which itself depends on the bit error rate (BER). The BER is computed according to the SNR and the technique of modulation (e.g., BPSK, FSK, OQPSK, etc.). Such radio link model is used, for example, in GloMoSim [2], WSNet [5], etc.

We consider a free-space pathloss model, a log-normal shadowing model and a rayleigh fading model with the same parameters described above. These models are evaluated according to three radio link models: (i) a threshold-based model; (ii) a BER-based model with a BPSK modulation; and (iii) a BER-based model with a OQPSK modulation. The obtained average number of connex components is shown on Figure 4.

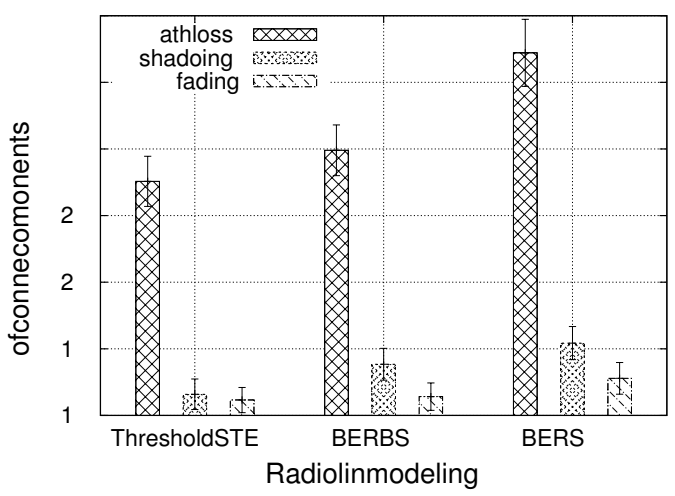

Figure 4: Impact of the radio link modeling on the average number of connex components.

From the results, shown on Figure 4, we can make two observations. First, we notice, quite obviously, that the radio range models impact the obtained average number of connex components. The shadowing and fading models induce a better network connectivity than the pathloss radio range model. Second, we observe that the radio link model impact also the network connectivity. Using a simple radio link model, such as the threshold-based model, yields better signal reception rates and thus a better network connectivity (i.e., a lower number of connex components). However, using more accurate models, such as the BER-based model, increases the packet loss and thus we get a lower network connectivity.

From these results it is clear that the radio link model have to be correctly chosen for an accurate performance evaluation of wireless systems. The use of simplistic radio link models is generally motivated by the scalability issue, however such models may leads to a better estimation of the channel conditions than more complex radio link models, and thus impacting the behavior of high level protocols. The use of accurate and precise radio link models is then a key point for the sake of realism and confidence in the simulation results.

\subsection{Effects of the interference modeling}

The previous subsections analyzed the impact of the radio range and the radio link modeling on a hello protocol without interference consideration. As interference is a major limiting factor for wireless communication systems, we investigate in what follows the impact of the interference modeling on the same above metrics. We consider the three radio range models introduced previously with a BER-based link model using a BPSK modulation scheme 


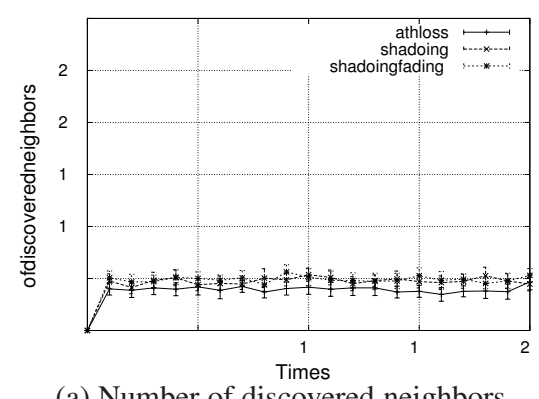

(a) Number of discovered neighbors.

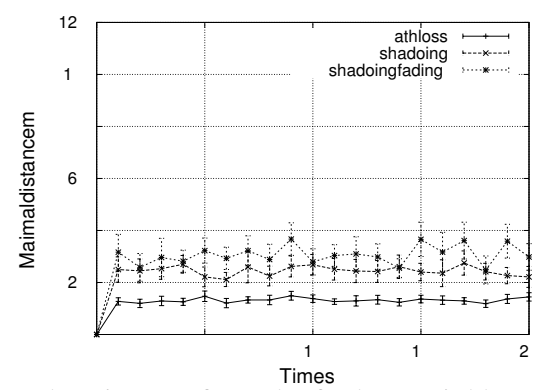

(b) Distance from the farthest neighbor

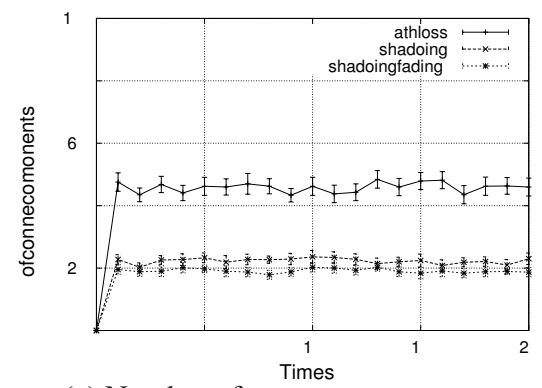

(c) Number of connex components.

Figure 5: Impact of the radio range modeling on the behavior of a hello protocol (with interference modeling).

and a full interference model with an adaptive SINR computation. The Figure 5 depicts the obtained average number of discovered neighbors, the distance from the farthest discovered neighbor and the number of connex components, in function of the time.

We notice on Figure 5 that the interference impacts strongly the obtained simulation results compared to the results shown on Figure 2. Hence, the average number of discovered neighbors decreases from 25 , for the case without interference, to 5 , with interference modeling. Moreover, we notice on Figure 5-(b) that the maximal distance from which a node may be discovered is also reduced. This fact is also verified on Figure 6.

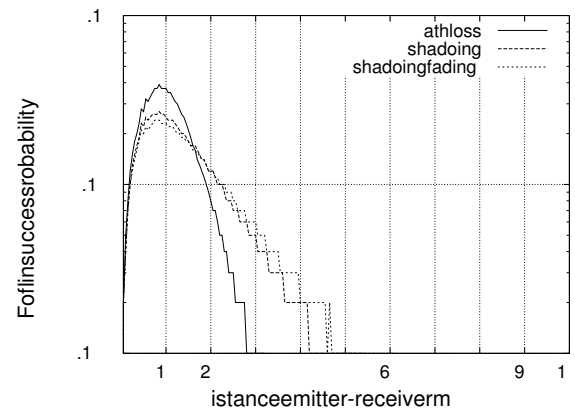

Figure 6: Impact of the radio range and the interference modeling on the link success probability.

Finally, regarding the average number of connex components shown on Figure 5-(c), the introduction of the interference modeling makes the network less connected, specially for the pathloss radio range model. Interference modeling is thus important during the evaluation of high level protocols as the behavior and the performance of these protocols depends on the PHY layer modeling accuracy. Evaluating the performance of protocols using inaccurate PHY layer modeling may affect the confidence of the simulation results.

We now investigate the interference modeling accuracy, focusing on the SINR computation methodology and the interference model. We perform the same set of simulations and we vary the interference modeling complexity. We consider a limited interference model with a maximal propagation range of $80 \mathrm{~m}$ and a full interference model. For both models, we use an adaptive and a cumulative SINR computation methodology. The impact of these models on the number of connex components is shown on Figure 8, and the graphs of connectivity obtained for the full interference model are shown on Figure 7.

Quite obviously, a better accuracy in the interference modeling

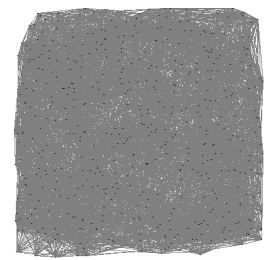

(a) avg degree $\approx 24$

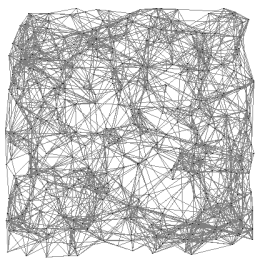

(b) avg degree $\approx 7$

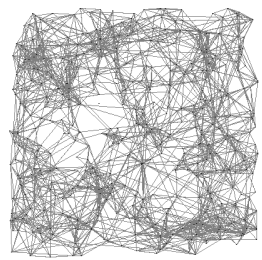

(c) avg degree $\approx 6$
Figure 7: The graphs of connectivity obtained with: (a) no interference consideration; (b) full interference model, BER+BPSK, cumulative SINR computation; and (c) full interference model, $B E R+B P S K$, adaptive SINR computation.

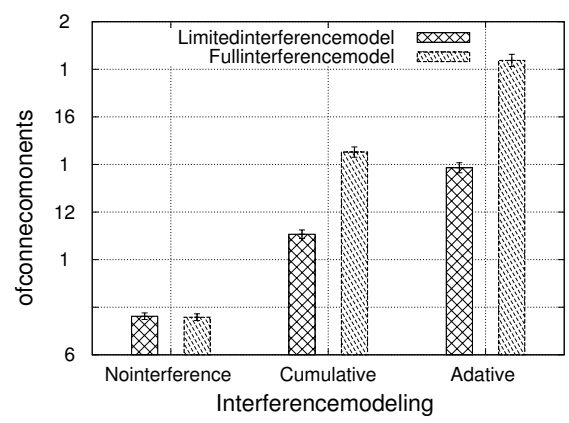

Figure 8: Impact of the SINR computation methodology on the number of connex components.

induces a loss of the network connectivity, or an increase of the number of connex components. As shown on Figure 8, the SINR computation methodology as well as the interference model induces a varying level of accuracy. If the limited interference model is generally motivated by the scalability issue, the use of the full interference model allows more accuracy and confidence in the simulation results, despite an increase of the computation overhead. The validity of the performance evaluation of high level protocols depends thus on the choices made for the PHY modeling, specially for the interference modeling.

\section{DESIGN ISSUES IN WIRELESS NETWORKS}

In this section, we analyze the effect of the hello protocol dimensioning and the battery power consumption on the behavior of application-layer protocols. These two issues are important, partic- 


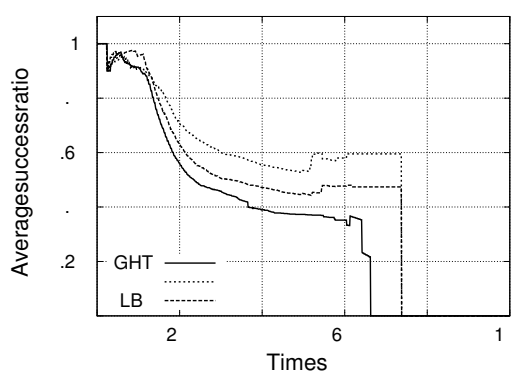

(a) 1 hello packet per $1 \mathrm{~s}$

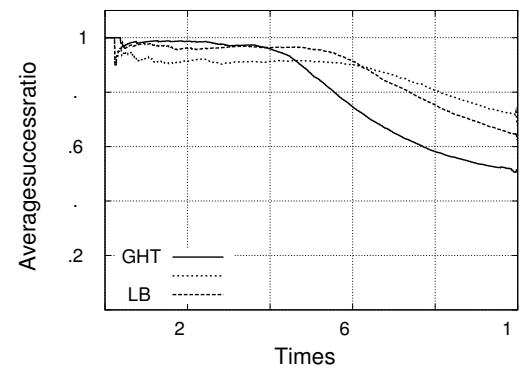

(b) 1 hello packet per $5 \mathrm{~s}$

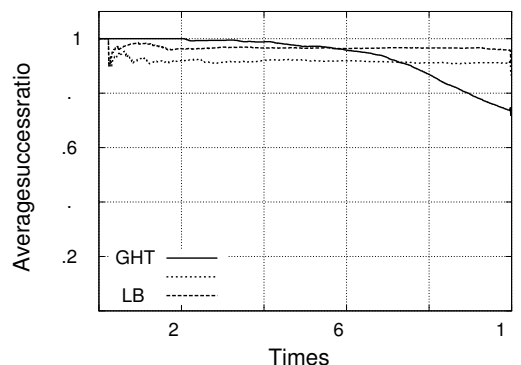

(c) 1 hello packet per $10 \mathrm{~s}$

Figure 9: Impact of the hello protocol dimensioning on the behavior of high level protocols.

ularly in the context of wireless sensor networks (WSN) where the energy is a major constraint and where most of the layer-3 protocols are based on a hello protocol. First, we present the assumptions as well as the application-layer protocols used for the analysis.Then we investigate the impact of the hello protocols and the battery model on the network lifetime and the behavior of some application-layer protocols.

\subsection{Assumptions}

We consider three application-layer data dissemination protocols: GHT [12], XY [13] and LBDD [7]. These protocols are used in the context of wireless sensor networks with mobile sinks for disseminating data reports from the sensors towards the base station (i.e., the sink). These protocols implements a rendez-vous area for storing the data reports generated by a source node. To collect the generated data, the sink sends its queries towards the rendezvous area and the corresponding data reports are sent back to the sink by geographic routing. The rendez-vous area can be a hashed location, as in GHT [12], or a central strip as in LBDD [7].

All these application-layer protocols are used on top of a greedy geographic routing which implements the basic hello protocol described in SubSection 4.1. Through a periodic exchange of hello packets, each node builds a neighbors table which is used during the routing process to forward messages from the sensor nodes to the sink. We consider 2000 static nodes deployed randomly over a $400 \times 400$ area, and a mobile sink moving according to a billiard mobility model. Each node runs a IEEE 802.15.4 868Mhz compliant radio with a transmission power of $0 \mathrm{dBm}$ and a radio sensitivity threshold of $-92 \mathrm{dBm}$, a IEEE 802.11 DCF MAC protocol, and a basic linear-decrease battery model. More realistic battery models can be considered [14] and yet have to be implemented.

We consider two metrics: (i) the success ratio: defined as the ratio of the total number of data packets received by the sink to the total number of packets generated by the sensors; and (ii) the number of active nodes: defined as the total number of active nodes in function of time. The first metric analyzes the performance of the application-layer protocols, while the latter reflects the network lifetime, as the number of active nodes decreases in function of time due to battery depletion.

\subsection{Effects of the Hello protocol dimensioning}

We first start by evaluating the impact of the hello protocol dimensioning on the behavior of GHT [12], XY [13] and LBDD [7]. We consider three hello protocol dimensioning: 1 hello packet transmission every 1, 5 and 10 seconds. The impact of this tuning on the network lifetime is shown on Figure 10, and the impact on the success ratio obtained by the application-layer protocols is shown on Figure 9.

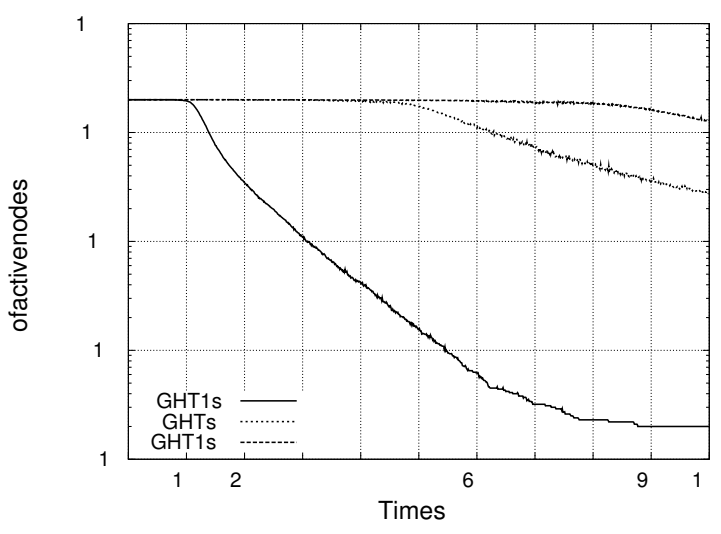

Figure 10: Impact of the hello periodicity on the network lifetime.

We observe, on Figure 10, that the hello protocol impacts significantly the network lifetime. In fact, the more the frequency of the hello packet transmission increases, the more the number of active nodes decreases in function of time. This is mainly due to the batteries depletion. This result highlights a clear tradeoff between the network lifetime and the effectiveness of protocols. If the use of a high frequency of hello-packet transmission reduces the network lifetime, it allows the application-layer protocols to be more effective in dynamic and lossy environments. A fine tuning of the hello protocol parameters if then important as it may impact the global performance of the network.

Regarding the behavior of the application-layer protocols, we notice on Figure 9 that the obtained success ratio vary in function of the hello protocol parameters. Indeed, we notice a high sensitivity of the application-layer protocols to the layer-3 parameters. For example, using a frequency of 1 hello packet per $1 \mathrm{~s}$, we notice that the success ratio of GHT decreases quickly compared to the other approaches. However, when using a frequency of 1 hello packet per $10 \mathrm{~s}$, we observe that the success ratio of GHT remains higher, compared to LBDD and XY. This raises again the importance of the dimensioning of layer-3 protocols during the performance evaluation of application-layer protocols.

\subsection{Effects of battery power consumption}

We now investigate the impact of the battery power consumption on the global performance evaluation. A correct dimensioning of the battery parameters is of great importance specially in the context of wireless sensor networks.

In the literature, this dimensioning is generally performed according to a given equipment standard, or simply randomly. Hence, 


\begin{tabular}{|c|c|c|}
\hline TX/RX ratio & Network lifetime (s) & Success ratio (\%) \\
\hline 0.5 & 480.168 & $0.185 \pm 0.06$ \\
\hline 0.75 & 557.811 & $0.248 \pm 0.07$ \\
\hline 1 & 617.277 & $0.273 \pm 0.07$ \\
\hline 1.5 & 399.811 & $0.292 \pm 0.07$ \\
\hline 2 & 272.877 & $0.368 \pm 0.1$ \\
\hline
\end{tabular}

Table 2: Impact of the battery power consumption on the global performance.

the transmission power is considered sometimes higher than the reception power, whereas in some simulation scenario it is considered equal or lower than the reception power. To better assess the impact of the battery power consumption on the global performance evaluation, we simulated the GHT protocol while varying the transmission power compared to the reception power. The impact of this dimensioning on the network lifetime and the obtained success ratio is depicted on Table 2. The results show that the dimensioning of the battery impact directly the global network lifetime as well as the performance of the application-layer protocol. Thus, if a protocol performs well with a given battery model, it can provides bad results with another battery model. Again, this highlights the importance of using detailed and accurate models for a precise performance evaluation of wireless systems.

\section{CONCLUSIONS}

Due to the complexity of wireless communication, simulation is generally the most convenient methodology for the performance evaluation of protocols. However, several factors may affect the confidence and the accuracy of the simulation results. These factors, which include the physical layer modeling and the dimensioning of protocol and environment parameters, increase the complexity of the performance evaluation.

Regarding the physical layer modeling, we have presented the PHY models implemented in five common simulators. Interference management, which is the main limiting factor for wireless systems, is probably the point where current simulators differ the most largely. Through intensive simulations, we have found that the physical layer, in particular interference management, impacts strongly the network connectivity as well as the behavior of networking protocols. For the sake of realism and confidence in simulation results, using accurate and detailed PHY models is thus a key point, despite an increase of the computation overhead. Moreover, we have found that the dimensioning of some protocol and environment parameters (i.e., the hello protocol and the battery model) is also important as it impacts the global performance of protocols. Thus, the question that arises is which parameters should we use when evaluating the performance of a protocol? and what simulation results must be considered as representative when comparing the performance of several protocols?. Since it is not possible to simulate a protocol with all the possible values of its parameters, we must either find the "good" values through a step of dimensioning, or by studying the possible existence of bounds for these parameters that would limit the performance of protocols and thus having a representative idea of its effectiveness. In general, it is a problem of representativeness of the obtained simulation results.

\section{REFERENCES}

[1] P. Almers and Al. Survey of channel and radio propagation models for wirelessmimo systems. EURASIP Journal onWireless Communications and Networking, 1:1-19, 2007.
[2] L. Bajaj, M. Takai, R. Ahuja, and R. Bagrodia. Simulation of large-scale heterogeneous communication systems. In Proceedings of MILCOM'99, November 1999.

[3] R. Barr, Z. J. Haas, and R. van Renesse. Scalable Wireless Ad hoc Network Simulation, chapter Ch. 19, pages pp. 297-311. Handbook on Theoretical and Algorithmic Aspects of Sensor, Ad hoc Wireless, and Peer-to-Peer Networks, 2005.

[4] D. Cavin, Y. Sasson, and A. Schiper. On the accuracy of manet simulators. In International Workshop on Principles of Mobile Computing (POMC), Toulouse, France, Oct. 2002. ACM.

[5] G. Chelius, A. Fraboulet, and E. Fleury. Worldsens: development and prototyping tools for application specific wireless sensors networks. In International Conference on Information Processing in Sensor Networks (IPSN), Boston, USA, April 2007.

[6] J.-M. Gorce, K. Jaffres-Runser, and G. de la Roche. A deterministic approach for fast simulations of indoor radio wave propagation. IEEE Transactions on Antennas and Propagation, 55(3,2):938-948, 2007.

[7] E. B. Hamida and G. Chelius. A line-based data dissemination protocol for wireless sensor networks with mobile sink. In Proceedings of the 2008 IEEE International Conference on Communications (ICC 2008), May 19-23.

[8] J. Heidemann, N. Bulusu, and J. Elson. Effects of detail in wireless network simulation. In Multiconference on Distributed Simulation. SCS, Jan. 2001.

[9] V. Naoumov and T. Gross. Simulation of large ad hoc networks. In Proceedings of MSWIM'03, pages 50-57, 2003.

[10] E. Ould-Ahmed-Vall, G. F. Riley, B. S. Heck, and D. Reddy. Simulation of large-scale sensor networks using gtsnets. In the 13th IEEE International Symposium on Modeling, Analysis, and Simulation of Computer and Telecommunication Systems (MASCOTS'05), 2005.

[11] P. P. Pham, S. Perreau, and A. Jayasuriya. New cross-layer design approach to ad hoc networks under Rayleigh fading. IEEE Journal on selected Areas in Communications, 23(1):28-39, 2005.

[12] S. Ratnasamy, B. Karp, S. Shenker, D. Estrin, R. Govindan, L. Yin, and F. Yu. Ght: A geographic hash table for data-centric storage in sensornets. In Proceedings of the First ACM International Workshop on Wireless Sensor Networks and Applications (WSNA'02), September 2002.

[13] Y. H. S. Das, H. Pucha. Performance comparison of scalable location services for geographic ad hoc routing. In Proceedings of IEEE INFOCOM 2005, pages 1228-1239, Miami, FL, March 13-17 2005.

[14] B. Staehle and D. Staehle. Impact of energy models on energy efficient sensor network routing. In Proceedings of the IEEE International Conference on Mobile Adhoc and Sensor Systems (MASS 2007), October 8-11 2007.

[15] M. Takai, J. Martin, and R. Bagrodia. Effects of wireless physical layer modeling in mobile ad hoc networks. In International Symposium on Mobile Ad Hoc Networking and Computing (MobiHoc), Long Beach, USA, Oct. 2001.

[16] M. Varshney and R. Bagrodia. Detailed models for sensor network simulations and their impact on network performance. In MSWiM, pages 70-77, 2004.

[17] Z. Wang and G. Giannakis. A simple and general parametrization quantifying performance in fading channels. IEEE trans on Communications, 51(8):1389-1398, August 2003. 